\title{
Tunable hysteresis effect for perovskite solar cells
}

\author{
Yaoguang Rong ${ }^{a \dagger}$, Yue Hu${ }^{a \dagger}$, Sandheep Ravishankar ${ }^{b \dagger}$, Huawei Liu ${ }^{a}$, Xiaomeng Hou $^{a}$, Yusong Sheng ${ }^{a}$, \\ Anyi Mei ${ }^{\mathrm{a}}$, Qifei Wang ${ }^{\mathrm{a}}$, Daiyu $\mathrm{Li}^{\mathrm{a}}, \mathrm{Mi} \mathrm{Xu}^{\mathrm{a}}$, Juan Bisquert ${ }^{\mathrm{b}, \mathrm{c}_{*}}$, Hongwei $\mathrm{Han}^{\mathrm{a} *}$ \\ Perovskite solar cells (PSCs) usually suffer an anomalous hysteresis in current-voltage measurements that leads to an \\ inaccurate estimation of the device efficiency. Although ion migration, charge trapping/detrapping and accumulation have \\ been proposed as a basis for the hysteresis, the origin of hysteresis has not been apparently unraveled. Herein we \\ reported a tunable hysteresis effect based uniquely on open-circuit voltage variations in printable mesoscopic PSCs with a \\ simplified triple-layer $\mathrm{TiO}_{2} / \mathrm{ZrO}_{2} /$ Carbon architecture. The electrons are collected by the compact $\mathrm{TiO}_{2} / \mathrm{mesoporous} \mathrm{TiO}_{2}$ (c- \\ $\mathrm{TiO}_{2} / \mathrm{mp}-\mathrm{TiO}_{2}$ ) bilayer, and the holes are collected by the carbon layer. By adjusting the spray deposition cycles for the c- \\ $\mathrm{TiO}_{2}$ layer, we achieved hysteresis-normal, hysteresis-free, and hysteresis-inverted PSCs. Such unique trends of tunable \\ hysteresis are analysed by considering the polarization of the $\mathrm{TiO}_{2} /$ perovskite interface, which can accumulate positive \\ charges reversibly. Successfully tuning the hysteresis effect clarifies the critical importance of the c-TiO ${ }_{2} /$ perovskite \\ interface in controlling the hysteretic trends observed, providing important insights towards the understanding of this \\ rapidly developing photovoltaic technology.
}

\section{Introduction}

Recently, organic-inorganic metal halide perovskite solar cells (PSCs) using a methylammonium lead halide $\left(\mathrm{MAPbX}_{3}, \mathrm{X}=\right.$ halogen) and its analogues as the light absorber have rapidly developed. ${ }^{1-3}$ These perovskites have a large absorption coefficient, high carrier mobility, long diffusion length and direct band gap, which make them ideal semiconductors for efficient photovoltaics. ${ }^{4-6}$ Besides, they are comprised of earthabundant materials and can be deposited by low-temperature solution methods, thus providing a promising prospect for applications. In the past several years, great efforts have been devoted to achieving high efficiency and developing beneficial device fabrication processes, launching the power conversion efficiencies (PCEs) to over $22 \%{ }^{7}$ At the same time, many attempts to understand the fundamental working principle and mechanism of PSCs have been reported, ${ }^{8-11}$ especially the anomalous current-voltage $(\mathrm{J}-\mathrm{V})$ hysteresis behavior. ${ }^{12-16}$ This anomalous hysteresis behaviour has been further complicated by the emergence of an 'inverted hysteresis' phenomenon recently. In general, 'normal' hysteresis is the case where there occurs a large loss of photocurrent and fill factor (FF) during the reverse to forward voltage (R-F) scan, (also leading to a reduced open-circuit voltage, $\left.V_{O C}\right)$ as compared to the forward to reverse ( $\mathrm{F}$ $\mathrm{R})$ scan. $^{14}$ Inverted hysteresis has been observed as a reduction in the short-circuit current $\left(J_{\mathrm{SC}}\right)$ for F-R scans compared to R-F scans by Shen et al. ${ }^{17}$ and Nemnes et al. ${ }^{18}$ while Tress et al. ${ }^{19}$ observed a loss in the FF in the form of an S-shaped kink for the F-R scan. Both

\footnotetext{
a. Michael Grätzel Center for Mesoscopic Solar Cells, Wuhan National Laboratory for Optoelectronics, School of Optical and Electronic Information, Huazhong University of Science and Technology, Wuhan 430074, Hubei, PR China. Email: hongwei.han@mail.hust.edu.cn

${ }^{b}$. Institute of Advanced Materials (INAM), Universitat Jaume I, 12006 Castelló, Spain.Email: bisquert@uji.es

Department of Chemistry, Faculty of Science, King Abdulaziz University, Jeddah, Saudi Arabia.

+ These authors contributed equally to this work.

Electronic Supplementary Information (ESI) available: [details of any supplementary information available should be included here]. See DOI: $10.1039 / x 0 \times x 00000 x$
}

normal and inverted hysteresis trends have been observed to become more acute for faster scans.

Since the observed trends in literature are mostly strong variations in the photocurrent, the general models employed to explain these types of hysteresis were based on variations in the collection efficiency of PSCs, which is modulated by the screening of the internal electric field by the mobile ions. ${ }^{14,20}$ Therefore, normal hysteresis was explained based on the fact that a forward bias voltage corresponded to a favourable charge extraction situation as compared to short-circuit, while this trend is reversed for inverted hysteresis due to a possible extraction barrier at the $\mathrm{TiO}_{2}$ /perovskite interface, where the accumulation of anions at short-circuit creates a dipole that facilitates band alignment and hence efficient charge extraction. ${ }^{19}$

Previously, we have developed a triple-layer architecture of $\mathrm{TiO}_{2} / \mathrm{ZrO}_{2} /$ Carbon to fabricate a hole-conductor-free printable mesoscopic PSC. ${ }^{21,22}$ By optimizing the mesoporous scaffold and perovskite absorber, hysteresis-less devices have been obtained with $\mathrm{TiO}_{2}$ as the electron transporting layer (ETL). ${ }^{23,24}$ In this study, it was found that the type of hysteresis effect in printable mesoscopic PSCs was highly dependent on the interlayer of $\mathrm{c}-\mathrm{TiO}_{2}$. Through controlling the deposition of c$\mathrm{TiO}_{2}$ layer, we realized normal and inverted hysteresis effect for such printable mesoscopic PSCs. Furthermore, the observed hysteresis was uniquely different from the typical hysteresis for PSCs as detailed previously, with the main difference in $J-V$ curves between forward and backward scans being the $V_{\mathrm{OC}}$, not $J_{\mathrm{SC}}$ or FF. We analysed the trends of such tunable hysteresis effect by considering the polarization of the 

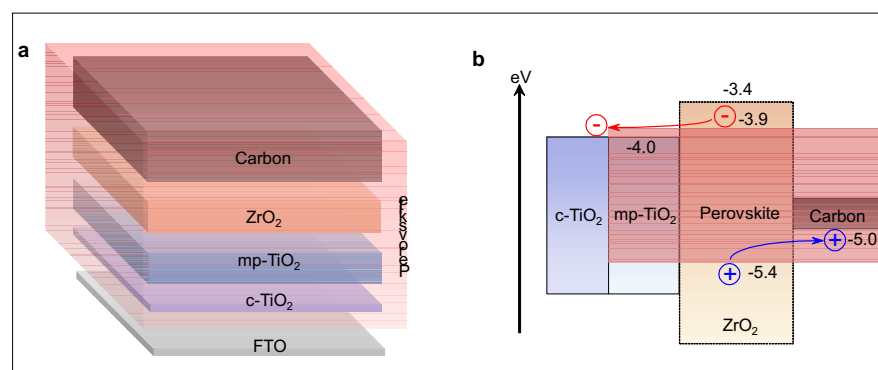

Fig. 1 Device configuration and corresponding working mechanism of a typical printable mesoscopic PSC. (a) Device architecture of FTO/c$\mathrm{TiO}_{2} / \mathrm{mp}-\mathrm{TiO}_{2} / \mathrm{ZrO}_{2} /$ Carbon/Perovskite. The perovskite of $(5-\mathrm{AVA})_{\times}(\mathrm{MA})_{1}$ ${ }_{x} \mathrm{Pbl}_{3}$ (5-AVA = 5-ammoniumvaleric acid) is infiltrated in the mesoporous layers of $\mathrm{mp}-\mathrm{TiO}_{2}, \mathrm{ZrO}_{2}$ and carbon by simple drop-casting method. (b) Band energy diagram of printable mesoscopic PSCs. The electron-hole pairs generated in the perovskite absorber separates by injecting the electron into $\mathrm{mp}^{-} \mathrm{TiO}_{2}$ and transporting the holes to the carbon electrodes.

$\mathrm{C}-\mathrm{TiO}_{2} /$ perovskite interface, and proposed this phenomenon is associated with the kinetics of accumulation and recombination of ions and charges at this interface during $\mathrm{J}-\mathrm{V}$ scans.

At present, there have been many reports of hysteresis-less PSCs that in cases have been obtained by combination of excellent bulk morphologies and manipulation of the contacts, often avoiding the use of metal-oxides. ${ }^{13,25,26}$ In this work, we show that robust devices fabricated with carbon hole extraction electrodes, still show a degree of dynamic hysteresis that is completely controlled by the quality of the thin $\mathrm{TiO}_{2}$ contact layer. We relate the extent of hysteresis observed in different conditions of dynamic scans and waiting times. Remarkably, the transient effects maintain the good features of photocurrent and the shape of $J-V$ curves, but affect mainly the apparent photovoltage. The results will be interpreted in terms of the interaction of charge accumulation and recombination effects, following previous observations of transient decays dependence on slow ionic kinetics. ${ }^{27}$ The analysis of photovoltage changes provides a general standpoint for understanding of the operation of contacts in the perovskite based photovoltaics.

\section{Results}

\subsection{Device configuration and working mechanism}

The device architecture used in this study is shown in Fig. $1 \mathrm{a}$, where $\mathrm{c}-\mathrm{TiO}_{2}, \mathrm{mp}-\mathrm{TiO}_{2}, \mathrm{ZrO}_{2}$ and carbon layers are deposited on a single FTO glass substrate layer by layer as the scaffold for depositing perovskite absorber. Typically, the three mesoporous layers have a thickness of $c a .1 .0,2.0$ and 10.0 $\mu \mathrm{m}$, respectively. A mixed-cation perovskite of $(5-A V A)_{x}(M A)_{1-}$ ${ }_{x} \mathrm{Pbl}_{3}$ (5-AVA $=5$-ammoniumvaleric acid) is infiltrated into the three mesoporous layers as the light absorber, and no HTM layer is needed in the device. The energy band alignment of the device (Fig. 1b) demonstrates that the electron-hole pairs generated in the perovskite absorber separates by injecting the electron into $\mathrm{mp}-\mathrm{TiO}_{2}$ and transporting the holes to the carbon back contact. ${ }^{22}$ In this process, the $\mathrm{ZrO}_{2}$ layer effectively prevents the direct contact between $\mathrm{TiO}_{2}$ and carbon layer. The $\mathrm{c}-\mathrm{TiO}_{2}$ layer prevents the valence band holes in the perovskite from reaching the FTO-covered front electrode. With the construction of these selective contacts, the electrons generated in the perovskite absorber are effectively collected by the ETL of the $\mathrm{mp}-\mathrm{TiO}_{2}$ and transferred to FTO front electrode via the $\mathrm{c}-\mathrm{TiO}_{2}$, and the holes are collected by the carbon back contact. The $\mathrm{mp}-\mathrm{TiO}_{2}$ layer has been considered mainly responsible for the hysteresis in conventional structured PSCs. ${ }^{13,25}$ Particularly, the thickness, pore size and treatment process of the $\mathrm{mp}-\mathrm{TiO}_{2}$ layer have been reported to influence the hysteresis of the devices. ${ }^{28-30}$ Here, for printable mesoscopic PSCs using a 1- $\mu \mathrm{m}$-thick $\mathrm{mp}$ $\mathrm{TiO}_{2}$ layer, hysteresis-less devices have been fabricated and reported. 22 We consider the $\mathrm{mp}-\mathrm{TiO}_{2}$ scaffold with extremely large surface area is able to provide efficient pathways for electron extraction and transportation rather than cause charge accumulation and possible hysteresis. On the contrary, for the $\mathrm{c}-\mathrm{TiO}_{2}$ layer that blocks hole injection from perovskite into FTO layer, it is critical to achieve a balance between recombination suppression and charge carrier transportation. We have found that the properties of the $\mathrm{c}-\mathrm{TiO}_{2}$ layer and corresponding interfaces significantly affect the $J-V$ hysteresis in the simplified triple-layer architecture based PSCs. For the deposition of $\mathrm{c}-\mathrm{TiO}_{2}$ layer, we firstly treated the FTO glass substrates with UV-Ozone for $2.5 \mathrm{~min}$, and then employed an aerosol spray pyrolysis method to prepare a c- $\mathrm{TiO}_{2}$ layer with different spraying cycles (Fig. S1, ESI+).
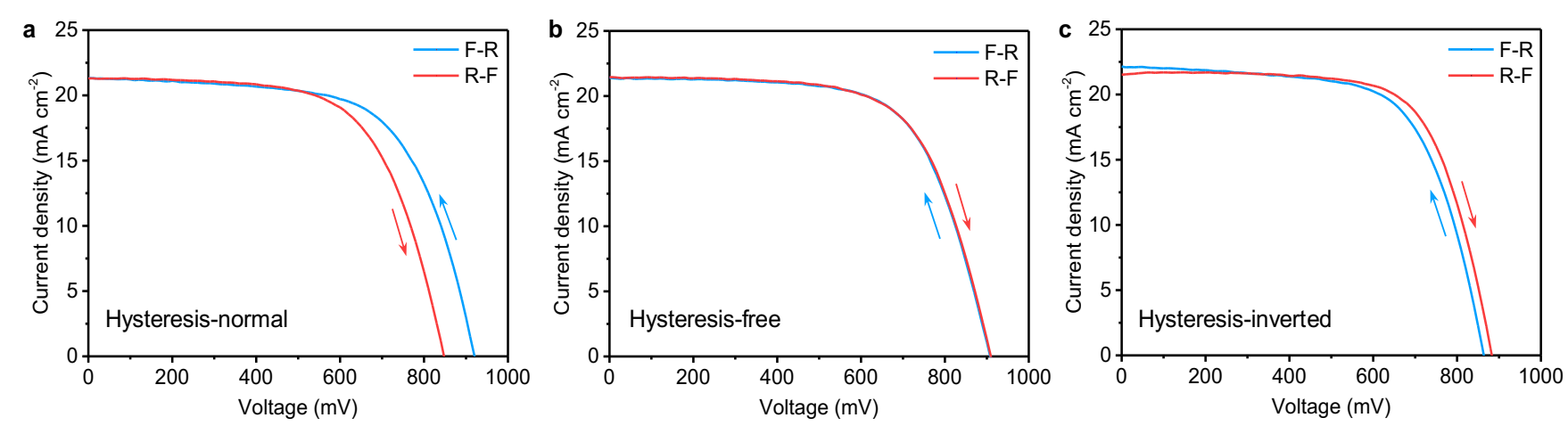

Fig. $2 \mathrm{~J}$-V curves of printable mesoscopic PSCs with different hysteresis behaviors. (a) Hysteresis-normal device that F-R scan shows better performance than R-F scan; (b) Hysteresis-free device that F-R scan and R-F scan show nondistinctive performance; (c) Hysteresis-inverted device that F-R scan shows slightly lower performance than R-F scan. (F-R: from forward bias to reverse bias; R-F: from reverse bias to forward bias; $s c a n$ rate $250 \mathrm{mV} \mathrm{s}^{-1}$ ). 

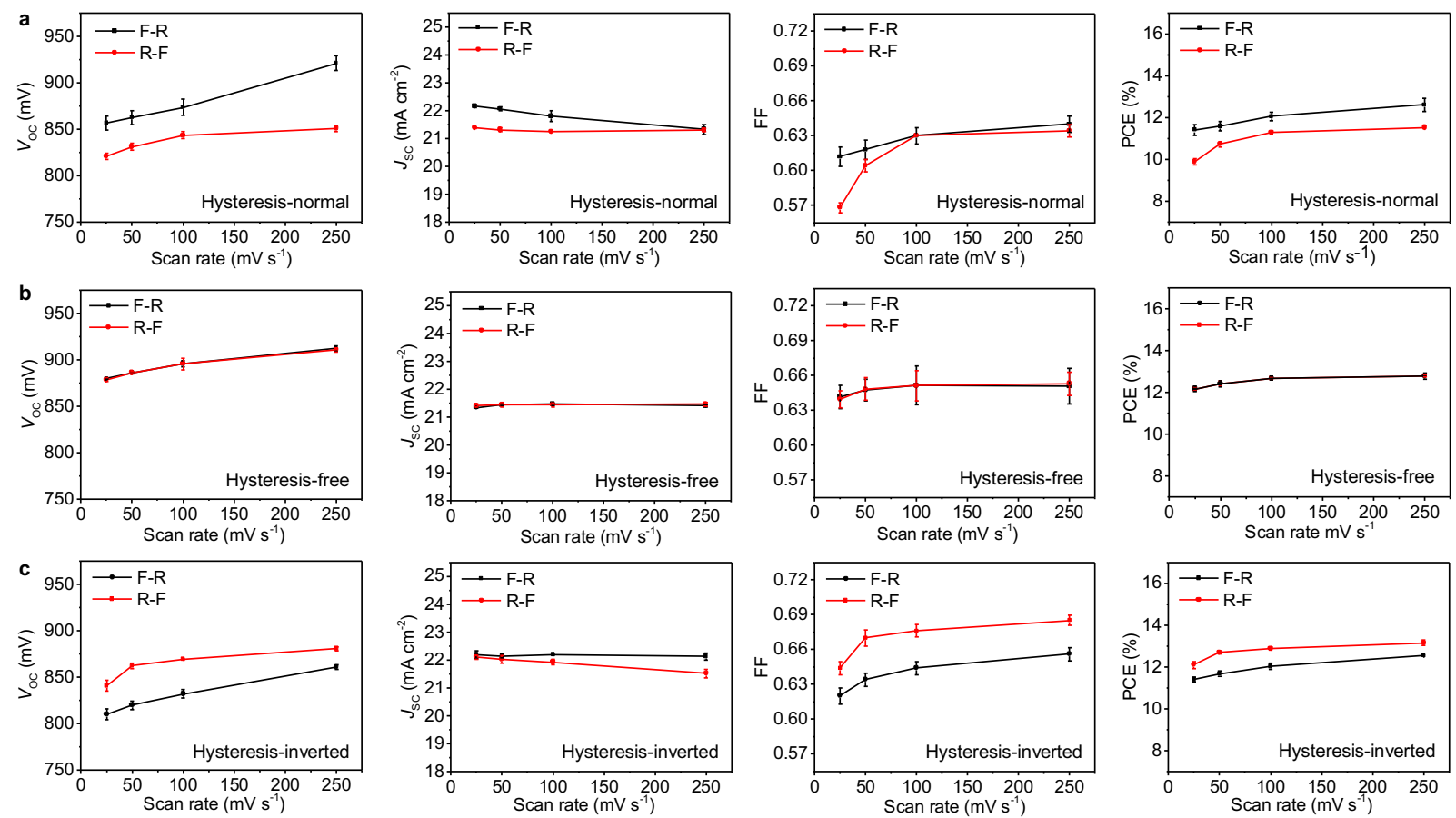

Fig. 3 Dependence of photovoltaic parameters on scan directions and scan rates. (a) hysteresis-normal, (b) hysteresis-free and (c) hysteresis-inverted printable mesoscopic PSCs.

\subsection{Hysteresis effect for printable mesoscopic PSCs}

To evaluate the hysteresis effect of the devices based on a c- $\mathrm{TiO}_{2}$ layer with different spray deposition cycles, $J-V$ curves were measured in forward scan direction (from reverse bias to forward bias, R-F) and backward scan direction (from forward bias to reverse bias, F-R) under simulated AM1.5 $100 \mathrm{~mW} \mathrm{~cm}^{-2}$ sunlight at a scan rate of $250 \mathrm{mV} \mathrm{s}^{-1}$. Note that the scan direction is indicated $\mathrm{R}-\mathrm{F}$ or F-R, and any waiting time at R or F bias is noted. Before $J-V$ scans, the devices were illuminated for several minutes, which can minimize the influence of the trap states and obtain a steady state. For typical $J-V$ hysteresis phenomenon in PSCs, the photocurrent and efficiency for F-R scan is higher than that for R-F scan. When 4 or 3 spraying cycles of $\mathrm{C}^{-\mathrm{TiO}_{2}}$ layer was applied for printable mesoscopic PSCs, the same trend was observed and we defined this case as hysteresis-normal (Fig. 2a). When we reduced the spraying cycles, it was found that the normal hysteresis effect could be firstly eliminated for 2 cycles (Fig. 2b), and then be inverted (Fig. 2c) completely for 1 cycle, which is regarded as tunable hysteresis effect for printable mesoscopic PSCs. The corresponding devices are coded as hysteresis-normal, hysteresis-free and hysteresis-inverted. Besides the spraying cycles of $\mathrm{c}-\mathrm{TiO}_{2}$ layer, such tunable hysteresis effect is also related to the UV-Ozone treatment (Table S1, ESI + ). It is important to remark that the dynamic hysteresis in our devices mainly affects the apparent photovoltage, and as a consequence the FF, which indicates that recombination changes occurring along the dynamic scan might play a dominant role in the observed changes. Further details on interpretation are presented in the last section of this work.

The photovoltaic parameters of devices showing different hysteresis effects are summarized in Table 1. Generally, the devices fabricated with different cycles sprayed $\mathrm{c}-\mathrm{TiO}_{2}$ layers show similar performance, especially for the $J_{S C}$ and PCE. When the spray deposition cycle was reduced from 4 to 1 , the $V_{\text {OC }}$ decreased from
$0.92 \mathrm{~V}$ to $0.86 \mathrm{~V}$, and the $\mathrm{FF}$ increased from 0.63 to 0.69 . For the parameters measured with F-R and R-F scans, the main differences lie in $V_{\text {OC }}$ rather than $J_{S C}$ or FF. This is quite different from the trend of typical hysteresis phenomenon for PSCs. ${ }^{12}$

Besides scan directions, the scan rates also influence the hysteresis behaviors of PSCs. To investigate the rate-dependent hysteresis effect of the devices, the photovoltaic parameters were measured at a scan rate of $25 \mathrm{mV} \mathrm{s}^{-1}, 50 \mathrm{mV} \mathrm{s}^{-1}, 100 \mathrm{mV} \mathrm{s}^{-1}$ and 250 $\mathrm{mV} \mathrm{s}^{-1}$, and compared in Fig. 3. Although the parameters measured at different scan rates varied, the differences between the values obtained with F-R and R-F scans still provide essential information for the hysteresis behaviors of printable mesoscopic PSCs. For hysteresis-normal devices (Fig. 3a), the difference in $V_{\text {oc }}$ for F-R and $\mathrm{R}-\mathrm{F}$ scans reduced from $\sim 70 \mathrm{mV}$ to $\sim 20 \mathrm{mV}$ when the scan rate decreased from $250 \mathrm{mV} \mathrm{s}^{-1}$ to $25 \mathrm{mV} \mathrm{s}^{-1}$, while the difference in $J_{\mathrm{SC}}$

Table 1 | Hysteresis effect, photovoltaic parameters and hysteresis effect index (HEI) of the devices fabricated using $\mathrm{c}^{-} \mathrm{TiO}_{2}$ layer with different spray deposition cycles.

\begin{tabular}{|c|c|c|c|c|c|c|c|}
\hline Hysteresis & c- $\mathrm{TiO}_{2}$ & Scan direction & $V_{\mathrm{OC}}(\mathrm{V})$ & $J_{\mathrm{SC}}\left(\mathrm{mA} \mathrm{cm}{ }^{-2}\right)$ & FF & PCE & HEI \\
\hline \multirow{2}{*}{ Normal } & \multirow{2}{*}{4 cycle } & F-R & 0.92 & 21.32 & 0.64 & 12.60 & \multirow{2}{*}{$0.0422 \pm 0.0068$} \\
\hline & & $\mathrm{R}-\mathrm{F}$ & 0.85 & 21.30 & 0.63 & 11.52 & \\
\hline \multirow{2}{*}{ Normal } & \multirow{2}{*}{3 cycle } & F-R & 0.92 & 21.40 & 0.63 & 12.37 & \multirow{2}{*}{$0.0171 \pm 0.0054$} \\
\hline & & R-F & 0.89 & 21.33 & 0.63 & 11.87 & \\
\hline \multirow{2}{*}{ Free } & \multirow{2}{*}{2 cycle } & F-R & 0.91 & 21.40 & 0.65 & 12.77 & \multirow{2}{*}{$0.0010 \pm 0.0035$} \\
\hline & & $\mathrm{R}-\mathrm{F}$ & 0.91 & 21.45 & 0.65 & 12.78 & \\
\hline \multirow{2}{*}{ Inverted } & \multirow{2}{*}{1 cycle } & F-R & 0.86 & 22.14 & 0.66 & 12.55 & \multirow{2}{*}{$-0.0434 \pm 0.0093$} \\
\hline & & $\mathrm{R}-\mathrm{F}$ & 0.88 & 21.51 & 0.69 & 13.15 & \\
\hline
\end{tabular}



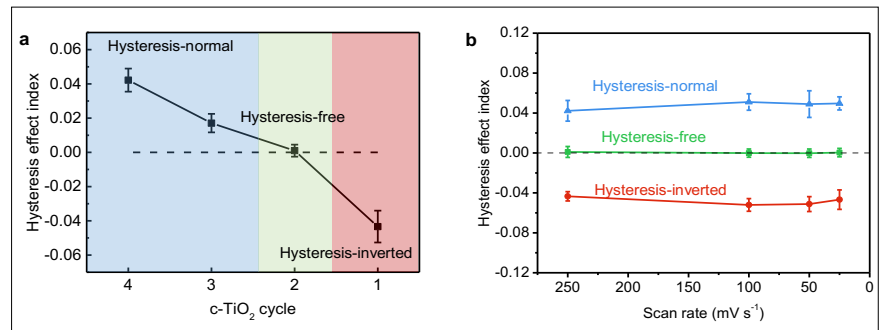

Fig. 4 Dependence of hysteresis effect index (HEI) (a) on $\mathrm{c}-\mathrm{TiO}_{2}$ layer spray deposition cycles and (b) on scanning rates for hysteresis-normal, hysteresis-free and hysteresis-inverted devices.

increased slightly. For the overall PCE, F-R at all scan rates led to a higher value than R-F. For hysteresis-free devices (Fig. 3b), F-R and $\mathrm{R}-\mathrm{F}$ scans showed almost the same photovoltaic parameters of $V_{\mathrm{OC}}$, $J_{S C}, F F$ and PCE at all different scan rates, though the values decreased slightly when the scan slowed down. For hysteresisinverted devices (Fig. 3C), R-F scan resulted in higher $V_{\mathrm{OC}}$ and FF while lower $J_{S C}$ than F-R scan, leading to higher PCE, and the difference between $\mathrm{R}-\mathrm{F}$ and $\mathrm{F}-\mathrm{R}$ scans did not change much for all the scan rates.

Series resistance $\left(R_{\mathrm{S}}\right)$ and shunt resistance $\left(R_{\mathrm{SH}}\right)$ of devices with different hysteresis behaviors are also summarized in Table S2 $(\mathrm{ESI}+)$ and compared in Figure S2 (ESI + ). All $R_{\mathrm{S}}$ slightly decreased as the scan rate increased from 50 to $250 \mathrm{mV} \mathrm{s}^{-1}$, while $R_{\mathrm{SH}}$ shows much more stable values as scan rates increased. Particularly, hysteresis-free devices showed slightly larger $R_{\mathrm{S}}$, but similar $R_{\mathrm{SH}}$, compared with hysteresis-normal and hysteresis-inverted devices independently of the scan rates. For $R_{\mathrm{S}}$ and $R_{\mathrm{SH}}$ measured at F-R and R-F scans, hysteresis-normal and hysteresis-inverted devices showed similar $R_{\mathrm{S}}$, but larger $R_{\mathrm{SH}}$ at $\mathrm{F}-\mathrm{R}$ scans. Hysteresis-free devices showed similar $R_{\mathrm{SH}}$, but smaller $R_{\mathrm{S}}$ at $\mathrm{F}-\mathrm{R}$ scans.

\subsection{Hysteresis index characterization}

A hysteresis index has been defined to describe the differences in $J-V$ curves appearing near open-circuit condition. ${ }^{28}$ In our case, since the hysteresis has been inverted causing the R-F scan to show higher current density and overall performance, a modified $\mathrm{J}-\mathrm{V}$ hysteresis effect index (HEI) is defined by equation 1 .

$$
\text { Hysteresis effect index }(\mathrm{HEI})=\frac{J_{R S\left(0.8 V_{o c}\right)}-J_{F S\left(0.8 V_{o c}\right)}}{\left(J_{R S\left(0.8 V_{o c}\right)}+J_{F S(0.8 V o c)}\right) / 2}
$$

where $J_{\mathrm{RS}(0.8 \mathrm{Voc})}$ and $J_{\mathrm{FS}\left(0.8 \mathrm{~V}_{\mathrm{OC}}\right)}$ represent current-density at $80 \%$ of $V_{\mathrm{OC}}$ for F-R and R-F scans, respectively. Even though our observations differ markedly for the early observations that lead to the definition of $\mathrm{HEI}$ in terms of current difference, ${ }^{15}$ the HEI shown in Fig. 4 still serves to illustrate the consistent evolution of $J-V$ curves depending on the changes of the $\mathrm{TiO}_{2}$ compact layer. For hysteresis-normal

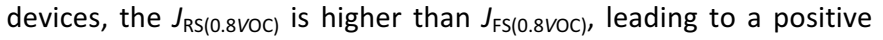
$\mathrm{HEI}$ value. On the contrary, hysteresis-inverted devices show negative $\mathrm{HEI}$ values, as shown in Table 1. The reduction of $\mathrm{c}-\mathrm{TiO}_{2}$ spray deposition cycles resulted in significant decrease of $\mathrm{HEI}$ from positive values of 0.0422 and 0.017 to 0.001 , then to a negative value of -0.0434 (Fig. 4a). The transition of HEl from hysteresisnormal to hysteresis-inverted is almost linear to the spray deposition cycles of $\mathrm{c}-\mathrm{TiO}_{2}$. Due to the relatively stable $J_{\mathrm{SC}}$ at different scan rates, the HEl values of all the three types of devices also stayed almost unchanged as the scan rate decreased (Fig. 4b). Considering that such printable mesoscopic PSCs show different trends for hysteresis effect, the variations in $V_{\text {OC }}$ provide more key information for analysing such tunable hysteresis for printable mesoscopic PSCs.

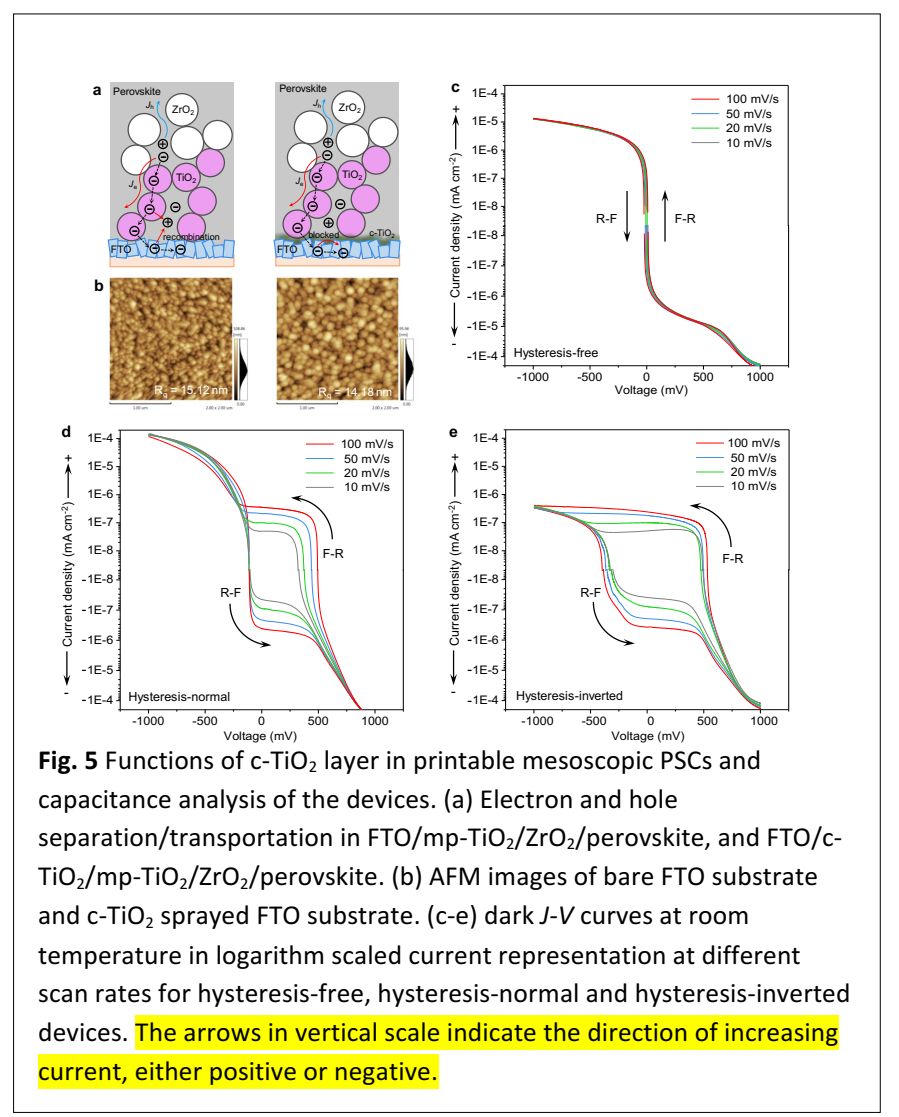

\subsection{Characterization of $\mathrm{c}-\mathrm{TiO}_{2}$ layer}

Ion migration with the perovskite film, high trap-state density for charge carriers at the perovskite surface and unbalanced charge carrier transport have been considered as the origins of the hysteresis effect for PSCs. ${ }^{3,27,28}$ Great efforts have been made to develop deposition methods that are able to obtain high-quality perovskite crystals. Typically, perovskite layers with large grain size, uniform morphology and appropriate composition will possess low defect density and show restricted ion migrations, which enable hysteresis-free/less devices with various architectures. ${ }^{25,31,32}$ In our case, the formation of the perovskite crystals was firstly templated by 5-AVA cations with preferential growth in the normal direction, and then hosted by the scaffold of $\mathrm{TiO}_{2} / \mathrm{ZrO}_{2} /$ Carbon triple-layer. Thus, the hysteresis effect originating from the quality of perovskite crystals has been minimized. Considering the remarkable device stability under working conditions, we propose that the J-V hysteresis effect in our devices is mainly due to the charge accumulation at the interfaces rather than composition change in the perovskite crystals, which would cause severe performance degradation of the devices. ${ }^{33,34}$ In printable mesoscopic PSCs, the $\mathrm{mp}-\mathrm{TiO}_{2}$ collects and transfer the electrons as the ETL, while c- $-\mathrm{TiO}_{2}$ layer works as a selective contact layer that transports electrons but blocks holes. Without a c- $-\mathrm{TiO}_{2}$ layer, the perovskite absorber will directly contact FTO front electrode, resulting in significant charge recombination and voltage loss for the device (Fig. 5a). Unfortunately, the electron mobility of $\mathrm{TiO}_{2}$ is only about $10^{-4} \mathrm{~cm}^{2} \mathrm{~V}$ ${ }^{1} \mathrm{~s}^{-1},{ }^{35,36}$ which is much lower than the transportation of charge carriers in $\mathrm{MAPbl}_{3}\left(10^{-2}-10^{0} \mathrm{~cm}^{2} \mathrm{~V}^{-1} \mathrm{~s}^{-1}\right)$. $^{37}$ Thus, it was proposed that the $\mathrm{mp}-\mathrm{TiO}_{2}$ layer in conventional structured PSCs would limit the electron transport, and cause capacitive hysteresis. ${ }^{38,39}$ Herein we show that the hysteresis in $\mathrm{c}-\mathrm{TiO}_{2}$ and $\mathrm{mp}-\mathrm{TiO}_{2}$ based PSCs is 

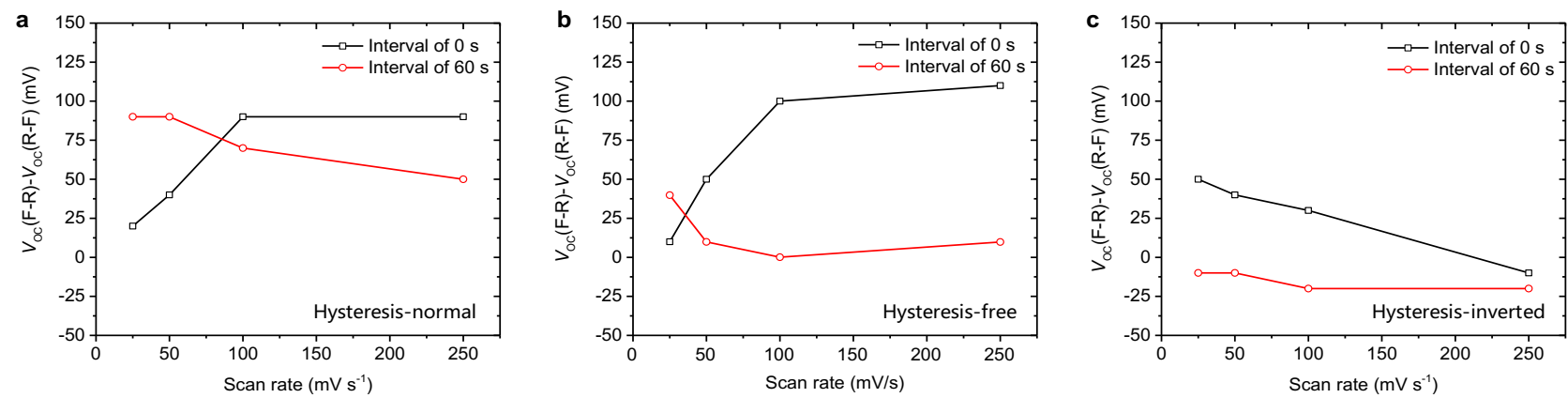

Fig. 6 Dependence of $\left[V_{o c}(F-R)-V_{o c}(R-F)\right]$ on scan rates. The hysteresis (a) -normal, (b) -free and (c) -inverted devices were continuously scanned with an interval of $0 \mathrm{~s}$ or discontinuously scanned with an interval of $60 \mathrm{~s}$ at $250 \mathrm{mV} \mathrm{s}^{-1}, 100 \mathrm{mV} \mathrm{s}^{-1}, 50 \mathrm{mV} \mathrm{s}^{-1}$ and $25 \mathrm{mV} \mathrm{s}^{-1}$ firstly in F-R direction, and then in R$F$ direction. The $V_{o c}$ were summarized and compared as $\left[V_{o c}(F-R)-V_{o c}(R-F)\right]$ versus scan rates.

mainly dependent on the $\mathrm{c}-\mathrm{TiO}_{2}$ layer and associated interfaces, not on the $\mathrm{mp}-\mathrm{TiO}_{2}$ layer.

As we show later, the $\mathrm{c}-\mathrm{TiO}_{2}$ surface meets the perovskite layer and here the ionic and electronic carriers find a barrier and establish essential charge accumulation and bandbending that when combined with surface recombination rates play a key role in the apparent voltage in transient conditions. In addition, the accumulation of electrons at the thin $\mathrm{c}^{-} \mathrm{TiO}_{2}$ itself is also an important factor to control the measured voltage. These elements will form the main considerations in our model, which motivates further analysis of the structure of the $\mathrm{c}-\mathrm{TiO}_{2}$ layer.

It should be noted that the deposition of $\mathrm{c}-\mathrm{TiO}_{2}$ layer between $\mathrm{mp}-\mathrm{TiO}_{2}$ and FTO layers also improve the contact and facilitate the charge transfer at the interfaces. Atomic force microscope (AFM) and scanning electron microscope (SEM) measurements were performed to unravel the differences of the surface morphology of bare and $\mathrm{c}-\mathrm{TiO}_{2}$ sprayed FTO substrates (Fig. $5 \mathrm{~b}$ and Fig. S3 $\uparrow$ ). The FTO substrate possessed a polyhedron composed morphology with a root-mean-square (RMS) roughness of $15.12 \mathrm{~nm}$. After spraying a c- $\mathrm{TiO}_{2}$ layer, the surface turned to be composed of sphere-shape particles and the roughness decreased slightly to $14.18 \mathrm{~nm}$. Due to the rough surface of FTO layer, we cannot accurately obtain the thickness of the $\mathrm{c}-\mathrm{TiO}_{2}$ layer sprayed on FTO substrates with 1 to 4 cycles. Instead, we sprayed the c- $\mathrm{TiO}_{2}$ layer on a silicon wafer with 8 cycles, and measured its thickness by a profilometer. The thickness of the $\mathrm{c}-\mathrm{TiO}_{2}$ layer was $52.7 \pm 5.50 \mathrm{~nm}$. Thus, we suppose the thickness of $\mathrm{c}-\mathrm{TiO}_{2}$ layers sprayed with $1-4$ cycles was $6.59 \pm 0.69 \mathrm{~nm}$, $13.18 \pm 1.37 \mathrm{~nm}, 19.76 \pm 2.06 \mathrm{~nm}$ and $26.35 \pm 2.75 \mathrm{~nm}$, respectively. It is expected that the FTO particles are wrapped by an ultra-thin $\mathrm{TiO}_{2}$ film, and this can be regarded as an interface modification rather than formation of an extra interlayer.

XPS measurements were carried out to investigate the Ti element in the 1-4 cycle $\mathrm{c}-\mathrm{TiO}_{2}$ sprayed FTO substrates. The Ti peak areas were summarized and compared using Sn peak as a reference peak. The $\mathrm{Ti} / \mathrm{Sn}$ ratio increased significantly from 1.581 to 3.93 , then to 10.3 and 22.1 for $1-4$ cycle c- $\mathrm{TiO}_{2}$ spraying (Fig. S4, ESI+). In addition, the work function of the $\mathrm{c}-\mathrm{TiO}_{2}$ sprayed FTO substrates were characterized, which decreased slightly from $4.79 \mathrm{eV}$ for the bare FTO to $4.45-4.62 \mathrm{eV}$ for the 1-4 cycle c- $\mathrm{TiO}_{2}$ sprayed FTO (Fig. S5, $\left.\mathrm{ESI}+{ }^{+}\right)$. The influence of the $\mathrm{c}-\mathrm{TiO}_{2}$ layer on the charge extraction was investigated by time-resolved photoluminescence (TRPL) spectra (Fig. S6, ESI $)$ ). Semi-devices of $\mathrm{FTO} / \mathrm{c}-\mathrm{TiO}_{2} / \mathrm{mp}-\mathrm{TiO}_{2} / \mathrm{ZrO}_{2} /$ Perovskite were fabricated with 1-4 cycles sprayed $\mathrm{c}-\mathrm{TiO}_{2}$ layer. A very fast decay followed by a slower decay was observed for all samples, as also observed elsewhere. ${ }^{40}$ The curves were fitted by a biexponential decay function, and the results are summarized in Table S3. It was found that $\tau_{1}$ and $\tau_{2}$ are almost identical as the c$\mathrm{TiO}_{2}$ spraying cycles increased, indicating thicker $\mathrm{c}-\mathrm{TiO}_{2}$ layer does not significantly modify the charge extraction of the $\mathrm{c}-\mathrm{TiO}_{2} / \mathrm{mp}-\mathrm{TiO}_{2}$ bilayer. Remove ref 42

\subsection{Capacitive hysteresis characterization}

Capacitance analysis of voltage sweep under dark was performed to investigate the capacitive hysteresis effect in printable mesoscopic PSCs (Fig. 5c-e), which was proposed to be caused by the charge accumulation at the $\mathrm{TiO}_{2} /$ perovskite interface. ${ }^{38,39}$ The voltage variation, $\Delta V$, applied to the solar cell produces a transient response before reaching the steady state. The time interval $\Delta t$ between applied voltage steps might be shorter than the time of relaxation. In the cases in which charging process occurs, an additional transient current is expected to be observed caused by the time variation of the accumulated charges. Furthermore, capacitive currents should vanish as the scan rate approaches steady-state conditions. For the hysteresis-normal device (Fig. 5d), significant capacitive hysteresis was observed along with similar dark current density with hysteresis-free and hysteresis-inverted devices at a forward (positive) bias. When spraying cycles for $\mathrm{c}^{-} \mathrm{TiO}_{2}$ layer decreased from 4 to 1 , the capacitive hysteresis effect firstly vanished (Fig. 5c), and then came back (Fig. 5e). The dark capacitive trends indicate a strong polarization may exist at the c$\mathrm{TiO}_{2} /$ perovskite interface, ${ }^{38}$ which can have a significant impact on the hysteretic trends under illumination. 


\subsection{Effect of delay time}

As mentioned in the previous section, for the hysteresis effect of printable mesoscopic PSCs, the main difference between F-R and R$F$ scans lies in $V_{\text {OC }}$ rather than $J_{S C}$ or FF. Thus, we summarized and compared the $V_{O C}$ for F-R and R-F scans, presented as $\left[V_{O C}(F-R)-V_{O C}\right.$ $(\mathrm{R}-\mathrm{F})$ ] versus scan rates in Fig. 6 . Notably, we found the intervals and sequence between $J-V$ scans also influence the device performance, which can provide us with some key information. In this case the $V_{\mathrm{OC}}$ was measured by sequential scans with interval of $0 \mathrm{~s}$ or $60 \mathrm{~s}$ at $250 \mathrm{mV} \mathrm{s}^{-1}, 100 \mathrm{mV} \mathrm{s}^{-1}, 50 \mathrm{mV} \mathrm{s}^{-1}$ and $25 \mathrm{mV} \mathrm{s}^{-1}$ firstly in $\mathrm{F}-\mathrm{R}$ direction, and then in R-F direction. The dependence of $V_{O C}$ on scan rates is shown in Fig. S7 (ESIt).

Without any delay (interval of $0 \mathrm{~s}$ ) between scans, $\left[V_{O C}(\mathrm{~F}-\mathrm{R})-V_{\mathrm{OC}}\right.$ $(\mathrm{R}-\mathrm{F})$ ] decreased as the scan rate slowed down from $250 \mathrm{mV} \mathrm{s}^{-1}$ to $25 \mathrm{mV} \mathrm{s}^{-1}$ for hysteresis-normal and hysteresis-free devices, while increased for the hysteresis-inverted device. Remarkably, in this condition, the hysteresis-inverted device showed higher $V_{O C}$ and PCE for F-R scans. In other words, this hysteresis-inverted device showed normal hysteresis effect. When an interval of $60 \mathrm{~s}$ was applied between the scans, the evolution of the $V_{\text {OC }}$ changed significantly. For hysteresis-normal and hysteresis-free devices, $\left[V_{\mathrm{OC}}(\mathrm{F}-\mathrm{R})-\mathrm{V}_{\mathrm{OC}}(\mathrm{R}-\mathrm{F})\right]$ increased as the scan rate slowed down, which showed completely opposite trends against the results obtained with interval of $0 \mathrm{~s}$. Significantly, the values of $\left[V_{\text {oc }}(F-R)-V_{o c}(R-F)\right]$ for hysteresis-inverted device became negative, and was consistent with the results discussed in the previous section.

\section{Discussion}

Since the $V_{O C}$ is the main photovoltaic variable modified in the type of hysteresis in our cells, the mechanisms of hysteresis cannot be explained based on the models of charge collection presently dominant in the literature. The observation of a consistent $J_{\mathrm{SC}}$ irrespective of scan rate and direction indicates that charge transport and extraction is identical at low $F$ biases, pointing to a preferential recombination mechanism at large $F$ biases, modifying the output voltage. In consideration of the above arguments, we explain the trends observed by considering the dominant contribution of the $\mathrm{c}-\mathrm{TiO}_{2} /$ perovskite interface. Fig. 7 provides a picture of the charge accumulation and polarization features at the interface. The formation of an accumulation of cations and electronic holes at the $\mathrm{c}^{-\mathrm{TiO}_{2}} /$ perovskite interface under illumination has been discussed ${ }^{42}$ and is shown in Fig. 7c. Note that the morphology of the mesoporous layer is neglected as it is assumed that rather narrow band bending occurs at the perovskite side. This accumulation of cations and electronic holes in a stabilized situation creates excess potential at the $\mathrm{c}-\mathrm{TiO}_{2} /$ perovskite interface that enhances $V_{\mathrm{OC}}$. It also serves as a strong source of surface recombination, by electrons coming from the $\mathrm{TiO}_{2}$ side of the contact.

Slow kinetic features result in hysteresis patterns that are mainly induced by the sluggish ionic dynamics, as already remarked on the capacitive response in Fig. 5 . The ionic displacement is completed at F voltage at the start of an F-R scan, causing the large accumulation, Fig. $7 c$, which is almost a steady-state value that is not modulated by ionic drift rates. Contrarily, for the R-F scan, the accumulation is delayed, which creates an asymmetry of $V_{\text {oc }}$ values. In an R-F scan, the ions are moved away at $R$ bias, so that the voltage cannot reach the same value as that of the F-R scan, explaining the normal hysteresis. It is noted from Fig. 2 that the F-R $V_{O C}$ is identical for (a)

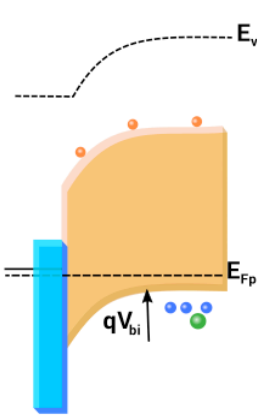

(b)

(c)

Fig. 7 Schematic diagram of the $\mathrm{c}-\mathrm{TiO}_{2}$ (blue layer)/perovskite (orange film) interface indicating the processes governing the $V_{\text {oc }}$. We consider a p-type perovskite at short circuit condition shown in (a) with a built-in potential at the perovskite/c- $-\mathrm{TiO}_{2}$ interface (electrons represented in red, holes and cations in blue and green respectively). (b) The PSC under an applied forward bias. Process 1 indicates the kinetics of drift of cations and holes towards the interface. (c) The PSC at large forward bias $\left(V_{o c}\right)$. The accumulation of cations and holes at the interface creates an upward band bending which can be described by a surface voltage $\mathrm{V}_{\mathrm{s}}$ represented in 2 . These accumulated charges can act as a preferential zone for both recombination with electrons in the bulk (3) and in the $\mathrm{c}-\mathrm{TiO}_{2} / \mathrm{FTO}$ region. Recombination pathway 4 crucially dependent on the thickness of the $\mathrm{c}-\mathrm{TiO}_{2}$ layer, and is the dominant mechanism controlling recombination rates of accumulated charges and the output $V_{\mathrm{OC}}$ in a transient scan.

normal hysteresis and hysteresis-free devices but is reduced by 50 $\mathrm{mV}$ for the inverted hysteresis samples. This indicates that another major factor to consider in the variation of photovoltage is the amount of recombination, as commented before. For thinner c- $\mathrm{TiO}_{2}$ layer, the covering of FTO may be incomplete, facilitating recombination of surface electrons as indicated in Fig. 7c. (process 4). The R-F scan $V_{\text {oc }}$ is increased by $\sim 70 \mathrm{mV}$ for the hysteresis-free and inverted hysteresis devices. This is also attributed to the larger work function of the thin $\mathrm{C}^{-} \mathrm{TiO}_{2}$ layers (Fig. S5, ESI+), creating smaller built-in potential for the perovskite, which allows for larger accumulation at a given $\mathrm{F}$ voltage in the R-F scan. For the inverted hysteresis devices, the large R-F voltage is sustained likely due to the sluggish kinetics of the surface recombination indicated in (4) in Fig. 7c. The improved R-F $V_{\mathrm{OC}}$ values contribute to the hysteresisfree behavior, and in conjunction with the $V_{O C}$ drop in the $F-R$ direction for a very thin $\mathrm{c}-\mathrm{TiO}_{2}$ layer, yields the inverted hysteresis trend in our samples. TRPL analysis showed that the kinetics of charge extraction is rather similar in the different thickness cases so that differences of interfacial kinetics does not appear a significant factor controlling the changes of hysteresis pattern.

We now proceed to explain the rich variety of behaviors of the dependence of $V_{\mathrm{OC}}$ on the scan rates (Fig. 3) and waiting times (Fig. $\mathrm{S} 7, \mathrm{ESI}+$ ). For the F-R scan, the accumulated positive charges at the surface at steady state recombine immediately upon cycling the voltage. Therefore, faster scans provide a larger $V_{O C}$ in the $F-R$ direction. This is observed for all devices, irrespective of the type of hysteresis, Fig. 3. The effect of waiting time under illumination is identical, where the accumulation is enhanced due to the photogenerated carriers, providing larger output voltages in all devices except the hysteresis-inverted devices. This could occur due to the increased surface recombination of photogenerated carriers for very thin $\mathrm{C}-\mathrm{TiO}_{2}$ layers previously described. For the R-F scans, the $V_{\text {OC }}$ improves for faster scan rates since the positive ions do not 
have enough time to drift away from the $\mathrm{c}-\mathrm{TiO}_{2}$ layer, thereby improving the $V_{\text {OC. }}$. The effect of waiting time under illumination is for the photovoltage to drive positive ions towards the c- $-\mathrm{TiO}_{2}$, thereby facilitating accumulation and also enhanced $V_{\text {oc }}$ during the RF scan.

In summary, we observe that the interplay of slow dynamics of charge accumulation and changes of recombination rates explains the observed hysteresis patterns. Hysteresis in PSCs is regarded as a combination of processes inherent to the operation of this device and not a single effect that can be removed. However, high quality of materials and interface do minimize the influence of dynamic hysteresis, which mainly affects the photovoltage according to the scanning procedure.

\section{Conclusions}

We have identified a tunable hysteresis effect consisting of $V_{\text {OC }}$ variations where the type of hysteresis can be altered solely through modification of the $\mathrm{c}-\mathrm{TiO}_{2}$ layer. The trends of tunable hysteresis effect are considered here to be caused by the polarization of the $\mathrm{c}-\mathrm{TiO}_{2} /$ perovskite interface, and is related to the kinetics of accumulation of positive charges at this interface during $J-V$ scans, in combination with the extent of surface recombination. The nature and properties of the c$\mathrm{TiO}_{2}$ /perovskite interface are critical for the recombination rates of accumulated charges at this interface, which is the dominant mechanism controlling the hysteretic trends in the PSCs. The demonstration of such tunable hysteresis effect in printable mesoscopic PSCs shows that the properties of c$\mathrm{TiO}_{2}$ /perovskite interface are crucial for controlling the device performance metrics. We showed that hysteresis-free devices can be formed by adapting specific conditions of the contact from a broad range of possibilities, and that hysteresis-free devices may not coincide with optimal solar cell operation. Therefore, we suggest that understanding the phenomenon of hysteresis is necessary for a better control of PSCs materials and interfaces.

\section{Acknowledgements}

The authors acknowledge financial support from the National Natural Science Foundation of China (91433203, 61474049, 51502141, 21702069), the Ministry of Science and Technology of China (2015AA034601), the 111 Project (No. B07038), and the China Postdoctoral Science Foundation (2016M600588). We also acknowledge funding from MINECO of Spain under Project MAT2016-76892-C3-1-R and Generalitat Valenciana Project PROMETEOII/2014/020. S. R. acknowledges Generalitat Valenciana for the grant (GRISOLIA/2014/034).

\section{Notes and references}

1. A. Kojima, K. Teshima, Y. Shirai and T. Miyasaka, J. Am. Chem. Soc., 2009, 131, 6050-6051.

2. H.-S. Kim, C.-R. Lee, J.-H. Im, K.-B. Lee, T. Moehl, A. Marchioro, S.-J. Moon, R. Humphry-Baker, J.-H. Yum, J. E. Moser, M. Grätzel and N.-G. Park, Sci. Rep., 2012, 2, 591.

3. M. A. Green, A. Ho-Baillie and H. J. Snaith, Nat. Photonics, 2014, 8, 506-514.
4. J.-H. Im, C.-R. Lee, J.-W. Lee, S.-W. Park and N.-G. Park, Nanoscale, 2011, 3, 4088-4093.

5. G. Xing, N. Mathews, S. Sun, S. S. Lim, Y. M. Lam, M. Grätzel, S. Mhaisalkar and T. C. Sum, Science, 2013, 342, 344-347.

6. Q. Dong, Y. Fang, Y. Shao, P. Mulligan, J. Qiu, L. Cao and J. Huang, Science, 2015, 347, 967-970.

7. W. S. Yang, B.-W. Park, E. H. Jung, N. J. Jeon, Y. C. Kim, D. U. Lee, S. S. Shin, J. Seo, E. K. Kim, J. H. Noh and S. I. Seok, Science, 2017, 356, 1376.

8. C. Eames, J. M. Frost, P. R. F. Barnes, B. C. O'Regan, A. Walsh and M. S. Islam, Nat. Commun., 2015, 6, 7497.

9. H.-S. Kim, I. Mora-Sero, V. Gonzalez-Pedro, F. FabregatSantiago, E. J. Juarez-Perez, N.-G. Park and J. Bisquert, Nat. Commun., 2013, 4, 2242.

10. L. Bertoluzzi, R. S. Sanchez, L. Liu, J.-W. Lee, E. Mas-Marza, H. Han, N.-G. Park, I. Mora-Sero and J. Bisquert, Energy Environ. Sci., 2015, 8, 910-915.

11. V. Gonzalez-Pedro, E. J. Juarez-Perez, W.-S. Arsyad, E. M. Barea, F. Fabregat-Santiago, I. Mora-Sero and J. Bisquert, Nano Lett., 2014, 14, 888-893.

12. H. J. Snaith, A. Abate, J. M. Ball, G. E. Eperon, T. Leijtens, N. K. Noel, S. D. Stranks, J. T.-W. Wang, K. Wojciechowski and W. Zhang, J. Phys. Chem. Lett., 2014, 5, 1511-1515.

13. H.-S. Kim, I.-H. Jang, N. Ahn, M. Choi, A. Guerrero, J. Bisquert and N.-G. Park, J. Phys. Chem. Lett., 2015, 6, 4633-4639.

14. W. Tress, N. Marinova, T. Moehl, S. M. Zakeeruddin, M. K. Nazeeruddin and M. Gratzel, Energy Environ. Sci., 2015, 8, 995-1004.

15. R. S. Sanchez, V. Gonzalez-Pedro, J.-W. Lee, N.-G. Park, Y. S. Kang, I. Mora-Sero and J. Bisquert, J. Phys. Chem. Lett., 2014, 5, 2357-2363.

16. E. L. Unger, E. T. Hoke, C. D. Bailie, W. H. Nguyen, A. R. Bowring, T. Heumüller, M. G. Christoforo and M. D. McGehee, Energy Environ. Sci., 2014, 7, 3690-3698.

17. H. Shen, D. A. Jacobs, Y. Wu, T. Duong, J. Peng, X. Wen, X. Fu, S. K. Karuturi, T. P. White, K. Weber and K. R. Catchpole, J. Phys. Chem. Lett., 2017, 8, 2672-2680.

18. G. A. Nemnes, C. Besleaga, V. Stancu, D. E. Dogaru, L. N. Leonat, L. Pintilie, K. Torfason, M. Ilkov, A. Manolescu and I. Pintilie, J. Phys. Chem. C, 2017, 121, 11207-11214.

19. W. Tress, J. P. Correa Baena, M. Saliba, A. Abate and M. Graetzel, Adv. Energy Mater., 2016, 6, 1600396.

20. G. Richardson, S. E. J. O'Kane, R. G. Niemann, T. A. Peltola, J. M. Foster, P. J. Cameron and A. B. Walker, Energy Environ. Sci., 2016, 9, 1476-1485.

21. Z. Ku, Y. Rong, M. Xu, T. Liu and H. Han, Sci. Rep., 2013, 3, 3132.

22. A. Mei, X. Li, L. Liu, Z. Ku, T. Liu, Y. Rong, M. Xu, M. Hu, J. Chen, Y. Yang, M. Grätzel and H. Han, Science, 2014, 345, 295-298.

23. Y. Rong, X. Hou, Y. Hu, A. Mei, L. Liu, P. Wang and H. Han, Nat. Commun., 2017, 8, 14555.

24. Y. Rong, Z. Ku, A. Mei, T. Liu, M. Xu, S. Ko, X. Li and H. Han, J. Phys. Chem. Lett., 2014, 5, 2160-2164.

25. J. H. Heo, H. J. Han, D. Kim, T. K. Ahn and S. H. Im, Energy Environ. Sci., 2015, 8, 1602-1608.

26. D. Yang, X. Zhou, R. Yang, Z. Yang, W. Yu, X. Wang, C. Li, S. Liu and R. P. H. Chang, Energy Environ. Sci., 2016, DOI: 10.1039/C6EE02139E.

27. R. Gottesman, P. Lopez-Varo, L. Gouda, Juan A. JimenezTejada, J. Hu, S. Tirosh, A. Zaban and J. Bisquert, Chem, 2016, 1, 776-789. 
28. H.-S. Kim and N.-G. Park, J. Phys. Chem. Lett., 2014, 5, 29272934.

29. J. Shao, S. Yang, L. Lei, Q. Cao, Y. Yu and Y. Liu, Chem. Mater., 2016, 28, 7134-7144.

30. F. Zhang, W. Ma, H. Guo, Y. Zhao, X. Shan, K. Jin, H. Tian, Q. Zhao, D. Yu, X. Lu, G. Lu and S. Meng, Chem. Mater., 2016, 28, 802-812.

31. Y. C. Kim, N. J. Jeon, J. H. Noh, W. S. Yang, J. Seo, J. S. Yun, A. Ho-Baillie, S. Huang, M. A. Green, J. Seidel, T. K. Ahn and S. I. Seok, Adv. Energy Mater., 2016, 6, 1502104.

32. X. Li, D. Bi, C. Yi, J.-D. Décoppet, J. Luo, S. M. Zakeeruddin, A. Hagfeldt and M. Grätzel, Science, 2016, 353, 58-62.

33. M. Bag, L. A. Renna, R. Y. Adhikari, S. Karak, F. Liu, P. M. Lahti, T. P. Russell, M. T. Tuominen and D. Venkataraman, J. Am. Chem. Soc., 2015, 137, 13130-13137.

34. C. Li, S. Tscheuschner, F. Paulus, P. E. Hopkinson, J. Kießling, A. Köhler, Y. Vaynzof and S. Huettner, Adv. Mater., 2016, 28, 2446-2454.

35. P. Tiwana, P. Docampo, M. B. Johnston, H. J. Snaith and L. M. Herz, ACS Nano, 2011, 5, 5158-5166.

36. I. Abayev, A. Zaban, F. Fabregat-Santiago and J. Bisquert, physica status solidi (a), 2003, 196, R4-R6.

37. C. Wehrenfennig, G. E. Eperon, M. B. Johnston, H. J. Snaith and L. M. Herz, Adv. Mater., 2014, 26, 1584-1589.

38. O. Almora, I. Zarazua, E. Mas-Marza, I. Mora-Sero, J. Bisquert and G. Garcia-Belmonte, J. Phys. Chem. Lett., 2015, 6, 16451652.

39. G. Garcia-Belmonte and J. Bisquert, ACS Energy Letters, 2016, 1, 683-688.

40. T. Handa, D. M. Tex, A. Shimazaki, A. Wakamiya and Y. Kanemitsu, J. Phys. Chem. Lett., 2017, 8, 954-960.

41. G. Xing, B. Wu, S. Chen, J. Chua, N. Yantara, S. Mhaisalkar, N. Mathews and T. C. Sum, Small, 2015, 11, 3606-3613.

42. I. Zarazua, J. Bisquert and G. Garcia-Belmonte, J. Phys. Chem. Lett., 2016, 7, 525-528. 